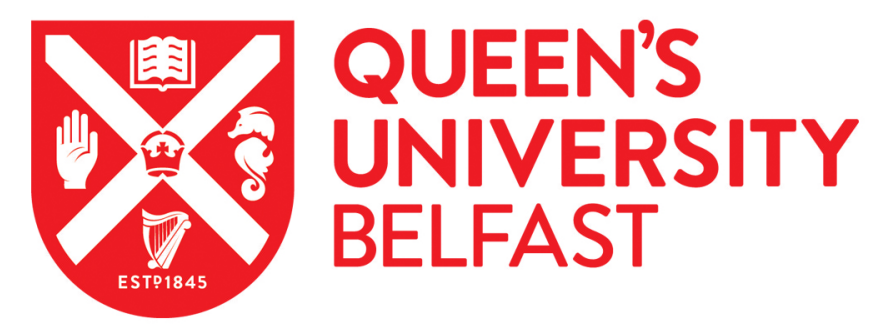

\title{
Construction of invariant polytopic sets with specified complexity
}

Athanasopoulos, N., Bitsoris, G., \& Lazar, M. (2014). Construction of invariant polytopic sets with specified complexity. International Journal of Control, 87(8), 1681-1693. https://doi.org/10.1080/00207179.2014.882518

Published in:

International Journal of Control

Document Version:

Peer reviewed version

Queen's University Belfast - Research Portal:

Link to publication record in Queen's University Belfast Research Portal

Publisher rights

Copyright 2014, Taylor \& Francis.

This work is made available online in accordance with the publisher's policies. Please refer to any applicable terms of use of the publisher.

\section{General rights}

Copyright for the publications made accessible via the Queen's University Belfast Research Portal is retained by the author(s) and / or other copyright owners and it is a condition of accessing these publications that users recognise and abide by the legal requirements associated with these rights.

Take down policy

The Research Portal is Queen's institutional repository that provides access to Queen's research output. Every effort has been made to ensure that content in the Research Portal does not infringe any person's rights, or applicable UK laws. If you discover content in the Research Portal that you believe breaches copyright or violates any law, please contact openaccess@qub.ac.uk. 
International Journal of Control

Vol. 00, No. 00, Month 200x, 1-21

\title{
RESEARCH ARTICLE
}

\section{Construction of Invariant Polytopic Sets with Specified Complexity}

\author{
Nikolaos Athanasopoulos ${ }^{a *}$, George Bitsoris ${ }^{b}$ and Mircea Lazar ${ }^{a}$ \\ ${ }^{a}$ Department of Electrical Engineering, Eindhoven University of Technology, P.O. Box 513, Eindhoven, \\ 5600 MB Netherlands; ${ }^{b}$ Control Systems Laboratory, Department of Electrical and Computer \\ Engineering, University of Patras, Eratosthenous 6, Rion 26500, Greece
}

(v3.5 released August 2008)

\begin{abstract}
The problem of construction of controlled invariant polytopic sets with specified complexity, for linear systems subject to linear state and control constraints, is investigated. First, geometric conditions for the enlargement of a polytopic set by adding a new vertex, in order to produce a polytopic set of specified complexity, are established. Next, conditions for such an enlargement of controlled invariant sets to preserve the controlled invariance property are presented. The established theoretical results are used to develop methods for the construction of admissible controlled invariant sets with specified complexity. Two numerical examples show how these results can be used for the computation of monotonic sequences of admissible controlled invariant sets of specified complexity.
\end{abstract}

Keywords: Controlled invariant sets, positively invariant sets, contractive sets, linear systems, constraints, complexity.

\section{Introduction}

A problem of interest in both the analysis and the design of linear systems in the presence of state and control constraints is the computation of the admissible domain of attraction for the autonomous case and of the admissible stabilizability region for the case of systems with inputs. For example, in model predictive control schemes, the computation of the controlled contractive region determines the domain of the state space where the convergence to the target set can be guaranteed. Also, the problem is relevant in many control applications, where the goal is to determine whether a desired set of initial states belongs to the admissible domain of attraction. Since controlled contractive, and as a consequence controlled invariant, subsets of the state space provide an approximation of the admissible domain of attraction, construction of an invariant set is one of the typical approaches to solve the aforementioned problem.

In numerous real-life applications state constraints are specified by linear inequalities that define bounded and closed polyhedral sets containing the origin in their interior. In this setting, several methods of construction of an admissible invariant polyhedral set for both continuoustime and discrete-time linear systems are available. These methods can be grouped in two categories, according to the approach used. The first category exploits the algebraic necessary and sufficient conditions of positive invariance and existence of Lyapunov functions, developed initially by (Molchanov and Pyatnitskii 1986a,b,c) for bounded polyhedral sets, by (Bitsoris 1988, 1991) for both bounded and unbounded polyhedral sets, by (Blanchini 1990) for polytopic sets under vertex representation, and extended later by (Hennet 1995, Polański 1995, Lazar 2010) and others. These conditions can be used directly to verify invariance of a given set. On the other

*Corresponding author. Email: n.athanasopoulos@tue.nl 
hand, exploiting them in order to construct an invariant set requires the analysis of the spectral properties of the system (Bitsoris 1988, 1991, Hennet 1995, Bobyleva and Pyatnitskii 2001), and leads to characterization of symmetric polyhedral positively invariant sets. The second category consists in computing convergent sequences of sets, that are mainly related to the backward reachability map and start from specific initial conditions (Bertsekas 1972, Gutman and Cwikel 1987, Cwikel and Gutman 1986, Aubin 1991, Gilbert and Tan 1991, Kolmanovsky, I. V. and Gilbert, E. G. 1998, Dórea and Hennet 1999, Blanchini et al. 1999, Raković and Fiacchini 2008). These methods provide polytopic approximations of the maximal controlled invariant set with any desired accuracy, but of non specificed complexity. Thus, although a number of works in the control research field deals with the characterization and computation of controlled invariant sets, there is little progress towards characterizing and constructing polytopic controlled invariant sets of bounded complexity and non-trivial size, except the works (Cannon et al. 2003, RohalIlkiv 2004, Pluymers et al. 2005, Blanchini et al. 2008, Benlaoukli and Olaru 2009, Blanco et al. 2010, Scibilia 2010, Scibilia et al. 2011) which utilize heuristic methods combined with special types of polytopic sets or the backward reachability map. Motivated by this lack of systematic constructive methods and the need to compute controlled invariant sets of low complexity, the goal of this article is to establish the theoretical foundations for developing design methods of construction of admissible controlled contractive polytopic sets with specified complexity. These methods can then be used for solving different kinds of constrained control problems where the complexity of the controlled contractive sets is considered as an additional constraint that must be respected. The main idea behind the approach consists in the addition of vertices to the convex hull of polytopic sets, resulting in conditions that can be easily verified by solving linear programming problems.

The paper is organized as follows. In Section 2, basic definitions and the problem statement are given. In Section 3, the theoretical framework for enlarging polytopic sets with specified complexity while preserving controlled invariance is established. In Section 4, these results are used for establishing algebraic necessary and sufficient conditions for such an enlargement method to produce admissible controlled invariant sets with specified complexity. Two illustrative examples of computation of monotone sequences of admissible polytopic sets are presented. The conclusions are drawn in Section 5. For clarity of exposition, the proofs of all theoretical results are presented in the Appendix.

\section{Problem statement}

Throughout the article, capital letters denote real matrices and lower case letters denote column vectors or scalars. $\mathrm{R}^{n}$ denotes the real $n$-space, $\mathrm{R}_{+}$denotes the set of nonnegative real numbers, $\mathrm{N}_{+}$denotes the set of nonnegative integers, $\mathrm{N}_{\left[q_{1}, q_{2}\right]}$ denotes the set of integers belonging to the interval $\left[q_{1}, q_{2}\right]$ and $\mathrm{R}^{n \times m}$ denotes the set of real $n \times m$ matrices.

The column and the row vectors of a matrix $G \in \mathrm{R}^{s \times n}$ are denoted by $g^{i}$ and $g_{i}^{\top}$ respectively,

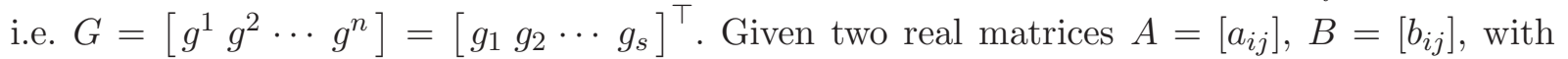
$A, B \in \mathrm{R}^{n \times m}$, the inequality $A \leq B(A<B)$ is equivalent to $a_{i j} \leq b_{i j}\left(a_{i j}<b_{i j}\right)$. Similar notation holds for vectors. The $p$-dimensional vector with all its elements equal to one is denoted by $e_{p}$, the $n \times m$ real matrix with all its elements equal to zero is denoted by $0_{n \times m}$ and the $n \times n$ identity is denoted by $I_{n \times n}$. Finally, given a subset $S \subset \mathrm{R}^{n}$ and a real number $r, r S$ denotes the set $r S:=\left\{y \in R^{n}:(\exists x \in S: y=r x)\right\}$.

The half-space representation of a convex polyhedral set having the origin as an interior point is defined by a vector inequality $G x \leq e_{p}, G \in \mathrm{R}^{p \times n}$ and is denoted by $\mathcal{P}(G)$, i.e.

$$
\mathcal{P}(G)=\left\{x \in \mathrm{R}^{n}: G x \leq e_{p}\right\}
$$

If the set $\mathcal{P}(G)$ is bounded then it is a polytope and can be equivalently defined as the convex 
hull of a set of vectors $v^{1}, v^{2}, . ., v^{q}$, namely

$$
\mathcal{Q}(\mathcal{V})=\operatorname{conv}\left(v^{1}, v^{2}, . ., v^{q}\right),
$$

where $\mathcal{V}=\left\{v^{1}, v^{2}, . ., v^{q}\right\}$. In such a description some of vectors $v^{1}, v^{2}, . ., v^{q}$, may be redundant. The minimal set of vectors $v^{i}$ required for defining a polytope constitutes the set of its vertices. In this paper the notation $\mathcal{Q}(\mathcal{V})$ will be used for describing polytopes having the origin as an interior point.

The complexity of a polytopic set can be characterized by the number of its vertices, the number of its faces or the structure of the induced face lattice (Ziegler 2007). In this article, the complexity of a polytopic set is defined as the number of its vertices.

We consider both continuous-time and discrete-time linear systems. The continuous-time systems are described by differential equations of the form

$$
\dot{x}(t)=A x(t)+B u(t),
$$

where $x \in \mathrm{R}^{n}$ is the state vector, $u \in \mathrm{R}^{m}$ is the input vector, $A \in \mathrm{R}^{n \times n}$ and $B \in \mathrm{R}^{n \times m}$ are the system and input matrices respectively and $t \in T_{c}$ is the time variable where $T_{c}=\mathrm{R}_{+}$. The discrete-time systems are described by difference equations of the form

$$
x(t+1)=A x(t)+B u(t),
$$

where the time variable $t \in T_{d}, T_{d}$ being the set of nonnegative integers $\mathrm{N}_{+}$. It will be assumed that the pair $(A, B)$ is stabilizable. Autonomous linear systems

$$
\dot{x}(t)=A x(t)
$$

and

$$
x(t+1)=A x(t)
$$

will also be considered as special cases of (1) and (2).

The state vector is constrained to belong to a bounded subset of the state space defined by a vector inequality of the form

$$
G_{x} x \leq e_{p_{x}}
$$

where $G_{x} \in \mathrm{R}^{p_{x} \times n}$. This means that the trajectories $x\left(t ; x_{0}\right)$ of the system are confined to lie within the polyhedral set $\mathcal{P}\left(G_{x}\right)$. Constraints are also imposed on the control input which has to satisfy linear inequalities of the form

$$
G_{u} u \leq e_{p_{u}}
$$

where $G_{u} \in \mathrm{R}^{p_{u} \times m}$.

Definition 2.1: Given system (1) ((2)), a set $\mathcal{S} \subset \mathrm{R}^{n}$ containing the origin as an interior point is said to be a controlled $\varepsilon$-contractive set with contraction factor $\varepsilon$ if and only if $\varepsilon>0$ $(0 \leq \varepsilon<1)$ and there exists a state-feedback control $u=f(x), f: \mathrm{R}^{n} \rightarrow \mathrm{R}^{m}$, such that for any initial state $x_{0} \in \mathcal{S}$ the corresponding trajectory $x\left(t ; x_{0}\right)$ of the resulting closed-loop system satisfies the relation $x\left(t ; x_{0}\right) \in e^{-\varepsilon\left(t-t_{0}\right)} \mathcal{S}$ for all $t_{0} \in T_{c}$ and $t \geq t_{0}\left(x\left(t ; x_{0}\right) \in \varepsilon^{\left(t-t_{0}\right)} \mathcal{S}\right.$ for all $x_{0} \in \mathcal{S}, t_{0} \in T_{d}$ and $\left.t \geq t_{0}\right)$.

Definition 2.2: Given system (1) ((2)) and constraints (5) and (6), a set $\mathcal{S} \subset \mathrm{R}^{n}$ containing the origin as an interior point is said to be an admissible controlled $\varepsilon$-contractive set with 
contraction factor $\varepsilon$ if and only if $\varepsilon>0(0 \leq \varepsilon<1)$ and there exists a state-feedback control $u=$ $f(x), f: \mathrm{R}^{n} \rightarrow \mathrm{R}^{m}$, such that for any initial state $x_{0} \in \mathcal{S}$ the corresponding trajectory $x\left(t ; x_{0}\right)$ of the resulting closed-loop system and the control input $f\left(x\left(t ; x_{0}\right)\right)$ respect the constraints (5) and (6) respectively for all $t \geq t_{0}$ and $x\left(t ; x_{0}\right) \in e^{-\varepsilon\left(t-t_{0}\right)} \mathcal{S}$, for all $t_{0} \in T_{c}$ and $t \geq t_{0}$ $\left(x\left(t ; x_{0}\right) \in \varepsilon^{\left(t-t_{0}\right)} \mathcal{S}\right.$ for all $x_{0} \in \mathcal{S}, t_{0} \in T_{d}$ and $\left.t \geq t_{0}\right)$.

If there exists an $\varepsilon$ satisfying the conditions of the above definition then the set $\mathcal{S}$ is said to be an admissible controlled contractive set. Finally, if the conditions in Definition 2.2 are satisfied by $\varepsilon=0$ for the continuous-time case and $\varepsilon=1$ for the discrete-time one, then the set $\mathcal{S}$ is said to be an admissible controlled invariant set.

Definition 2.3: Given the autonomous system (3) ((4)) and the state constraints (5), a set $\mathcal{S} \subset \mathrm{R}^{n}$ containing the origin as an interior point is said to be an admissible $\varepsilon$-contractive set with contraction factor $\varepsilon$ if and only if $\varepsilon>0(0 \leq \varepsilon<1)$ and for any initial state $x_{0} \in \mathcal{S}$ the corresponding trajectory $x\left(t ; x_{0}\right)$ of the resulting closed-loop system respects the state constraints (5) and satisfies the relation $x\left(t ; x_{0}\right) \in e^{-\varepsilon\left(t-t_{0}\right)} \mathcal{S}$ for all $t_{0} \in T_{c}$ and $t \geq t_{0}\left(x\left(t ; x_{0}\right) \in \varepsilon^{\left(t-t_{0}\right)} \mathcal{S}\right.$ for all $x_{0} \in \mathcal{S}, t_{0} \in T_{d}$ and $\left.t \geq t_{0}\right)$.

Remark 1: Positively invariant and controlled invariant sets are special cases of contractive and controlled contractive sets respectively with $\varepsilon=0$ for the continuous-time case and $\varepsilon=1$ for the discrete-time case. Thus, the exposition is made for the general case when $\varepsilon \geq 0(0 \leq \varepsilon \leq 1)$.

When the matrix pair $(A, B)$ of an unconstrained system (1) or (2) is stabilizable, it is possible to determine a controlled contractive polytope $\mathcal{Q}\left(\mathcal{V}^{0}\right)=\operatorname{conv}\left(v^{01}, v^{02}, \ldots, v^{0 q_{0}}\right)$ by computing a stabilizing linear state-feedback control law (Hennet 1995, Bobyleva and Pyatnitskii 2001). Then, by proper scaling, we can always determine a sufficiently "small" admissible controlled contractive polytope $\mathcal{Q}\left(r \mathcal{V}^{0}\right)=\operatorname{conv}\left(r v^{01}, r v^{02}, \ldots, r v^{0 q_{0}}\right)$, where $r \in \mathrm{R}_{+}$. Moreover, when the pair $(A, B)$ is controllable, it is always possible to construct a contractive symmetric polytope $\mathcal{Q}\left(\mathcal{V}_{o}\right)$ of desired complexity $q_{0} \geq 2^{n}$. Generally, the so obtained admissible controlled contractive sets cover a small volume of the maximal admissible set. On the other hand, the reachabilitybased methods (Bertsekas 1972, Gutman and Cwikel 1987, Cwikel and Gutman 1986, Aubin 1991, Gilbert and Tan 1991, Kolmanovsky, I. V. and Gilbert, E. G. 1998, Dórea and Hennet 1999, Blanchini et al. 1999, Raković and Fiacchini 2008) converge (under assumptions) to the maximal admissible controlled contractive set, however, the sets produced are usually of high complexity.

The problem investigated in this article has a different setting since not only the volume but also the complexity of the admissible controlled contractive set is included in the specifications. To this end, we determine the conditions for an enlarged polytope, which is obtained by adding a new vertex to the vertex representation of an admissible controlled contractive polytopic set, to preserve this property and, in addition, to be of a specified complexity. These general theoretical results are the tools that can be used for the development of methods of construction of admissible controlled contractive polytopic sets with specified complexity.

Thus, the problem to be investigated is formulated as follows: Given the system (1) ((2)), the state and input constraint sets (5) and (6) and an admissible controlled contractive set $\mathcal{Q}(\mathcal{V})$ of complexity $q$, find a systematic method of computing admissible controlled contractive supersets $\mathcal{Q}\left(\mathcal{V}^{*}\right)$ of a specified complexity $q^{*}$.

\section{Theoretical Foundations}

Perhaps the simplest method of enlarging an admissible controlled contractive polytopic set $\mathcal{Q}(\mathcal{V})=\operatorname{conv}\left(v^{1}, \ldots, v^{q}\right)$ is to add a new component $v^{*} \notin \mathcal{Q}(\mathcal{V})$ in its vertex description to obtain a new admissible controlled contractive polytopic set $\mathcal{Q}\left(\mathcal{V}^{*}\right)=\operatorname{conv}\left(v^{1}, \ldots, v^{q}, v^{*}\right)$. Then, $\mathcal{Q}(\mathcal{V}) \subset$ $\mathcal{Q}\left(\mathcal{V}^{*}\right)$ will hold by construction. In this subsection, we establish the necessary and sufficient 
conditions for such an enlargement approach to produce an admissible controlled contractive polytopic set of specified complexity.

\subsection{Enlargement of polytopes with specified complexity}

Addition of a new vertex $v^{*}$, situated outside a polytope $\mathcal{Q}(\mathcal{V})=\operatorname{conv}\left(v^{1}, v^{2}, \ldots, v^{q}\right)$ will generate an enlarged polytope $\mathcal{Q}(\mathcal{V})=\operatorname{conv}\left(v^{1}, \ldots, v^{q}, v^{*}\right)$ which has not necessarily higher complexity. The reason for this is that although $\mathcal{Q}\left(\mathcal{V}^{*}\right)$ is defined as the convex hull of more vectors than those used for the definition of the set $\mathcal{Q}(\mathcal{V})$, some of them may become redundant when $v^{*}$ is added. Consequently, the set $\mathcal{Q}\left(\mathcal{V}^{*}\right)$ may have equal or even lower complexity, depending on where the new vertex $v^{*}$ is located in the state space. In this subsection, we establish necessary and sufficient conditions for this enlargement procedure to produce polytopic sets with specified complexity.

Let $S \subset \mathrm{R}^{n}$ be a polytopic set with $q$ vertices and $p$ faces and with vertex and half-plane representations

$$
\mathcal{Q}(\mathcal{V})=\operatorname{conv}\left(v^{1}, \ldots, v^{q}\right)
$$

and

$$
\mathcal{P}(G)=\left\{x \in \mathrm{R}^{n}: G x \leq e_{p}\right\}
$$

respectively.

With each vertex $v^{k}, k \in \mathrm{N}_{[1, q]}$ of the polytope $\mathcal{S}=\mathcal{Q}(\mathcal{V})$, we associate the set of indices $N_{\mathcal{S}}\left(v^{k}\right) \subset \mathrm{N}_{[1, p]}$, defined by the relation

$$
N_{\mathcal{S}}\left(v^{k}\right):=\left\{j \in \mathrm{N}_{[1, p]}: g_{j}^{\top} v^{k}=1\right\}
$$

The set $N_{\mathcal{S}}\left(v^{k}\right)$ represents the set of indices $j$ which correspond to the faces $g_{j}^{\top} x=1$ of the polytope $\mathcal{S}=\mathcal{Q}(\mathcal{V})$ that pass through the vertex $v^{k}$. Moreover, with each vertex $v^{k}, k \in \mathrm{N}_{[1, q]}$ of the polytope $S=\mathcal{Q}(\mathcal{V})$, we associate the set $\mathcal{A}_{k}$, defined by the relation

$$
\mathcal{A}_{k}:=\left\{x \in \mathrm{R}^{n}: g_{j}^{\top} x \geq 1, \quad j \in N\left(v^{k}\right)\right\} .
$$

The sets $\mathcal{A}_{k}$ are polyhedral cones formed by the faces $g_{k}^{\top}=1$ of the polytope $\mathcal{S}=\mathcal{Q}(\mathcal{V})$ and point outside the set $\mathcal{S}$. The following theorem establishes conditions for the proposed enlargement approach not to produce a polytope of higher complexity. Recall that the complexity of the set is defined as the number of its vertices.

Theorem 3.1: Given a polytope $\mathcal{S} \subset \mathrm{R}^{n}$ with vertex and half-space representations (7) and (8) respectively, the polytope

$$
\mathcal{Q}\left(\mathcal{V}^{*}\right)=\operatorname{conv}\left(v^{1}, \ldots, v^{q}, v^{*}\right)
$$

satisfies the set relation

$$
\mathcal{Q}(\mathcal{V}) \subset \mathcal{Q}\left(\mathcal{V}^{*}\right)
$$

and is of equal or lower complexity than $\mathcal{Q}(\mathcal{V})$ if and only if $v^{*} \notin \mathcal{Q}(\mathcal{V})$ and

$$
v^{*} \in \mathcal{A}_{1} \cup \mathcal{A}_{2} \cup \ldots \cup \mathcal{A}_{q}
$$


Using this result we can establish conditions for the enlargement of a polytope by adding a new vertex to produce a new polytope with specified lower complexity.

Theorem 3.2: Given a polytope $\mathcal{S} \subset \mathrm{R}^{n}$ of complexity $q$ with vertex and half-space representations (7) and (8) respectively, the polytope

$$
\mathcal{Q}\left(\mathcal{V}^{*}\right)=\operatorname{conv}\left(v^{1}, \ldots, v^{q}, v^{*}\right)
$$

satisfies the set relation

$$
\mathcal{Q}(\mathcal{V}) \subset \mathcal{Q}\left(\mathcal{V}^{*}\right)
$$

and is of complexity lower or equal to $q^{*}=q-l, l \in \mathrm{N}_{[0, q-n-1]}$ if and only if $v^{*} \notin \mathcal{Q}(\mathcal{V})$ and there exist at least $l+1$ indices $k_{1}, k_{2}, \ldots, k_{l+1} \in \mathrm{N}_{[1, q]}$ such that

$$
\mathcal{A}_{k_{1}} \cap \mathcal{A}_{k_{2}} \cap \ldots \cap \mathcal{A}_{k_{l+1}} \neq \varnothing
$$

and

$$
v^{*} \in \mathcal{A}_{k_{1}} \cap \mathcal{A}_{k_{2}} \cap \ldots \cap \mathcal{A}_{k_{l+1}} .
$$

A direct consequence of Theorems 3.1, 3.2, which is of practical importance, follows.

Corollary 3.3: Given a polytope $\mathcal{S} \subset \mathrm{R}^{n}$ of complexity $q$ with vertex and half-space representations (7) and (8) respectively, the polytope

$$
\mathcal{Q}\left(\mathcal{V}^{*}\right)=\operatorname{conv}\left(v^{1}, \ldots, v^{q}, v^{*}\right)
$$

satisfies the set relation

$$
\mathcal{Q}(\mathcal{V}) \subset \mathcal{Q}\left(\mathcal{V}^{*}\right)
$$

and is of equal complexity to that of $Q(\mathcal{V})$ if and only if $v^{*} \notin \mathcal{Q}(\mathcal{V})$ and there exists an index $k \in \mathrm{N}_{[1, q]}$ such that

$$
v^{*} \in \mathcal{A}_{k} \backslash\left(\mathcal{A}_{1} \cup \mathcal{A}_{2} \cup \ldots \mathcal{A}_{k-1} \cup \mathcal{A}_{k+1} \cup \ldots \cup \mathcal{A}_{q}\right) .
$$

Example 3.4 The results established in this subsection enables one to partition the region outside a given polytope $S=\mathcal{Q}(\mathcal{V})$ in subregions where a new vertex $v^{*}$ must be situated for the enlarged polytope $\mathcal{Q}\left(\mathcal{V}^{*}\right)$ to be of specified complexity.

To show this graphically, we consider a polytopic set $S \subset \mathrm{R}^{2}$ with eight vertices, shown in Figure 1 in white color. We are interested in identifying the regions which correspond to different complexities of the set $\mathcal{Q}\left(\mathcal{V}^{*}\right)$ for the subset $\mathcal{X} \subset \mathrm{R}^{2}$ of the state space, which is the square of length 2. By calculating first the index sets $N_{\mathcal{S}}\left(v^{k}\right), k \in \mathrm{N}_{[1,8]}$ and next the sets $\mathcal{A}_{k} \cap \mathcal{X}, k \in \mathrm{N}_{[1,8]}$, application of Theorem 3.1, Theorem 3.2 and Corollary 3.3 yields the polytopic regions for which the complexity of the set $\mathcal{Q}\left(\mathcal{V}^{*}\right)$ is identified. Thus, as shown in Figure 1, the complexity of $\mathcal{Q}\left(\mathcal{V}^{*}\right)$ is increased by one for regions that are depicted with +1 , the complexity remains the same for the regions with 0 , and is reduced by one, two and three for the regions shown with $-1,-2$ and -3 respectively.

\section{$3.2 \quad$ Enlargement of contractive polytopes}

The results stated in the preceding subsection can be utilized to identify the regions of the state space where a new vertex can be added such that the resulting enlarged polytope has 


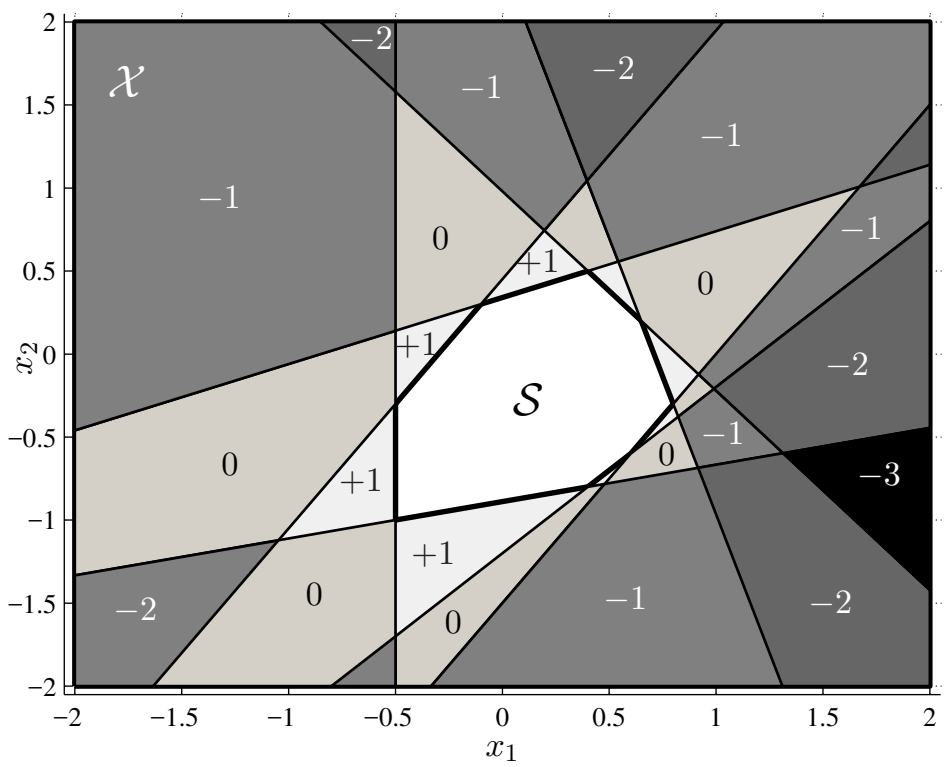

Figure 1. The polytopic sets $S=\mathcal{Q}(\mathcal{V})$ and $\mathcal{X}$, and the partition of the set $\mathcal{X} \backslash \mathcal{Q}(\mathcal{V})$ in regions according to the complexity of the resulting set when a vertex is added to the convex hull of $S$.

a desired complexity. In this subsection, we establish the additional conditions that must be satisfied for the enlarged polytope $\mathcal{Q}\left(\mathcal{V}^{*}\right)=\operatorname{conv}\left(v^{1}, \ldots, v^{q}, v^{*}\right)$ generated by adding a new vertex $v^{*}$ to a controlled $\varepsilon$-contractive or simply $\varepsilon$-contractive set $\mathcal{Q}(\mathcal{V})=\operatorname{conv}\left(v^{1}, \ldots, v^{q}\right)$, to be also a controlled $\varepsilon$-contractive or $\varepsilon$-contractive set (Athanasopoulos 2010, Athanasopoulos and Bitsoris 2010, Athanasopoulos et al. 2012). First, we consider the case of continuous-time systems (1).

Theorem 3.5: Given a controlled $\varepsilon$-contractive set $\mathcal{Q}(\mathcal{V})=\operatorname{conv}\left(v^{1}, \ldots, v^{q}\right)$ with respect to the continuous-time system (1) and a vector $v^{*} \in \mathrm{R}^{n}$, the polytope $\mathcal{Q}\left(\mathcal{V}^{*}\right)=\operatorname{conv}\left(v^{1}, \ldots, v^{q}, v^{*}\right)$ is a controlled $\varepsilon$-contractive set if and only if there exist vectors $u^{*} \in \mathrm{R}^{m}, p^{*} \in \mathrm{R}^{q}$ and a scalar $p_{q+1}^{*}$, such that

$$
\begin{aligned}
A v^{*}+B u^{*} & =V p^{*}+p_{q+1}^{*} v^{*}, \\
e_{q}^{\top} p^{*}+p_{q+1}^{*} & \leq-\varepsilon, \\
p^{*} & \geq 0,
\end{aligned}
$$

where $V=\left[\begin{array}{lll}v^{1} & v^{2} \cdots v^{q}\end{array}\right]$.

Corollary 3.6: Given an $\varepsilon$-contractive set $\mathcal{Q}(\mathcal{V})=\operatorname{conv}\left(v^{1}, \ldots, v^{q}\right)$ with respect to the continuous-time system (3) and a vector $v^{*} \in \mathrm{R}^{n}$, the polytope $\mathcal{Q}\left(\mathcal{V}^{*}\right)=\operatorname{conv}\left(v^{1}, \ldots, v^{q}, v^{*}\right)$ is an $\varepsilon$-contractive set if and only if there exists a vector $p^{*} \in \mathrm{R}^{q}$ and a scalar $p_{q+1}^{*}$, such that

$$
\begin{aligned}
A v^{*} & =V p^{*}+p_{q+1}^{*} v^{*}, \\
e_{q}^{\top} p^{*}+p_{q+1}^{*} & \leq-\varepsilon, \\
p^{*} & \geq 0,
\end{aligned}
$$

where $V=\left[\begin{array}{lll}v^{1} & v^{2} \cdots v^{q}\end{array}\right]$.

Next, we consider the case of discrete-time systems.

Theorem 3.7: Given a controlled $\varepsilon$-contractive set $\mathcal{Q}(\mathcal{V})=\operatorname{conv}\left(v^{1}, \ldots, v^{q}\right)$ with respect to 
the discrete-time system (2) and a vector $v^{*} \in \mathrm{R}^{n}$, the polytope $\mathcal{Q}\left(\mathcal{V}^{*}\right)=\operatorname{conv}\left(v^{1}, \ldots, v^{q}, v^{*}\right)$ is a controlled $\varepsilon$-contractive set if and only if there exist vectors $u^{*} \in \mathrm{R}^{m}, p^{*} \in \mathrm{R}^{q}$ and a scalar $p_{q+1}^{*}$, such that

$$
\begin{aligned}
A v^{*}+B u^{*} & =V p^{*}+p_{q+1}^{*} v^{*}, \\
e_{q}^{\top} p^{*}+p_{q+1}^{*} & \leq \varepsilon \\
p^{*} & \geq 0 \\
p_{q+1}^{*} & \geq 0
\end{aligned}
$$

where $V=\left[\begin{array}{lll}v^{1} & v^{2} \cdots v^{q}\end{array}\right]$.

Corollary 3.8: Given an $\varepsilon$-contractive set $\mathcal{Q}(\mathcal{V})=\operatorname{conv}\left(v^{1}, \ldots, v^{q}\right)$ with respect to the discrete-time system (4) and a vector $v^{*} \in \mathrm{R}^{n}$, the polytope $\mathcal{Q}\left(\mathcal{V}^{*}\right)=\operatorname{conv}\left(v^{1}, \ldots, v^{q}, v^{*}\right)$ is an $\varepsilon$-contractive set if and only if there exists a vector $p^{*} \in \mathrm{R}^{q}$ and a scalar $p_{q+1}^{*}$, such that

$$
\begin{aligned}
A v^{*} & =V p^{*}+p_{q+1}^{*} v^{*}, \\
e_{q}^{\top} p^{*}+p_{q+1}^{*} & \leq \varepsilon, \\
p^{*} & \geq 0, \\
p_{q+1}^{*} & \geq 0,
\end{aligned}
$$

where $V=\left[\begin{array}{llll}v^{1} & v^{2} \cdots & v^{q}\end{array}\right]$.

\section{Admissible controlled contractive sets with specified complexity}

We are now in a position to establish necessary and sufficient conditions for the enlargement of a polytopic admissible controlled contractive set while respecting complexity specifications. Let $\mathcal{Q}(\mathcal{V})=\operatorname{conv}\left(v^{1}, \ldots, v^{q}\right)$ be an admissible controlled $\varepsilon$-contractive set of the continuous-time system (1) with state and input constraints (5) and (6), of complexity $q$ and with half-space representation $\mathcal{P}(G), G \in \mathrm{R}^{p \times n}$. The main result of this section for continuous-time systems (1) follows.

Theorem 4.1: Given a complexity $q^{*}$ and a vector $v^{*} \in \mathrm{R}^{n}$, the set $\mathcal{Q}\left(\mathcal{V}^{*}\right)=$ $\operatorname{conv}\left(v^{1}, \ldots, v^{q}, v^{*}\right)$ is an admissible controlled $\varepsilon$-contractive set of complexity $q^{*}$ for the continuous-time system (1) if and only if there exist vectors $u^{*} \in \mathrm{R}^{m}, p^{*} \in \mathrm{R}^{q}$, a scalar $p_{q+1}^{*} \in \mathrm{R}$ and indices $i=1,2, \ldots, q-q^{*}+1$ satisfying the algebraic relations

$$
\begin{aligned}
A v^{*}+B u^{*} & =V p^{*}+v^{*} p_{q+1}^{*} \\
p^{*} & \geq 0 \\
e_{q}^{\top} p^{*}+p_{q+1}^{*} & \leq-\varepsilon \\
G_{x} v^{*} & \leq e_{p_{x}} \\
G_{u} u^{*} & \leq e_{p_{u}} \\
g_{j}^{\top} v^{*} & >1, \quad \forall j \in N_{\mathcal{S}}\left(v^{k_{i}}\right),
\end{aligned}
$$

where $V=\left[v^{1} v^{2} \cdots v^{q}\right]$.

A similar result, established for discrete-time systems, follows. 
Theorem 4.2: Given a complexity $q^{*}$ and a vector $v^{*} \in \mathrm{R}^{n}$, the set $\mathcal{Q}\left(\mathcal{V}^{*}\right)=$ $\operatorname{conv}\left(v^{1}, \ldots, v^{q}, v^{*}\right)$ is an admissible controlled $\varepsilon$-contractive set of complexity $q^{*}$ for the discretetime system (2) if and only if there exist vectors $u^{*} \in \mathrm{R}^{m}, p^{*} \in \mathrm{R}^{q}$, a scalar $p_{q+1}^{*} \in \mathrm{R}$ and indices $i=1,2, \ldots, q-q^{*}+1$ satisfying the algebraic relations

$$
\begin{aligned}
A v^{*}+B u^{*} & =V p^{*}+v^{*} p_{q+1}^{*} \\
p_{q+1}^{*} \geq 0, \quad p^{*} & \geq 0 \\
e_{q}^{\top} p^{*}+p_{q+1}^{*} & \leq \varepsilon \\
G_{x} v^{*} & \leq e_{p_{x}} \\
G_{u} u^{*} & \leq e_{p_{u}} \\
g_{j}^{\top} v^{*} & >1 \quad \forall j \in N_{\mathcal{S}}\left(v^{k_{i}}\right),
\end{aligned}
$$

where $V=\left[v^{1} v^{2} \cdots v^{q}\right]$.

Remark 2: Similar results are obtained for the autonomous case if relation (33) of Theorem 4.1 and relation (39) of Theorem 4.2 are substituted by

$$
A v^{*}=V p^{*}+v^{*} p_{q+1}^{*}
$$

The importance of Theorems 4.1 and 4.2 and of the corresponding results for the autonomous case lies in the fact that from a given or determined admissible controlled $\varepsilon$-contractive polytopic set $\mathcal{Q}(\mathcal{V})=\operatorname{conv}\left(v^{1}, \ldots, v^{q}\right)$, we can construct an enlarged admissible controlled $\varepsilon$-contractive polytopic set $\mathcal{Q}\left(\mathcal{V}^{*}\right)=\operatorname{conv}\left(v^{1}, \ldots, v^{q}, v^{*}\right)$ with specified complexity. This is possible by solving the set of algebraic relations (33)-(38) for the continuous-time case and (39)-(44) for the discrete-time case for different sets of integers $\left\{k_{1}, k_{2}, \ldots, k_{q-q^{*}+1}\right\}$. It is worth noting that the relations (33)-(38) and (39)-(44) are linear except the product in the right-hand side of equations (33),(39) between the sign-definite ${ }^{1}$ scalar $p_{q+1}^{*}$ and the vector $v^{*}$. Thus, considering $p_{q+1}^{*}$ as a scalar parameter, these algebraic relations are linear with respect to the unknown variables $v^{*}, u^{*}, p_{q}^{*}$.

An approach to the determination of the unknown variables $v^{*}, u^{*}, p_{q}^{*}$ is to define and solve an optimization problem having (33)-(38) or (39)-(44) as constraints. If a linear optimization criterion is chosen, then this optimization problem can be solved by applying any standard scalar-parameterized mixed-integer linear programming approach (Schrijver 1998). For example, solving the optimization problem

$$
\min _{v^{*}, u^{*}, p_{q}^{*}, p_{q+1}^{*}}\left\{e_{q}^{\top} p^{*}+p_{q+1}^{*}\right\}
$$

under constraints (33)-(38) for the continuous-time case and under constraints (39)-(44) for the discrete-time case, we determine a new vertex $v^{*}$ making the enlarged polytope $\mathcal{Q}(\mathcal{V})=$ $\operatorname{conv}\left(v^{1}, \ldots, v^{q}, v^{*}\right)$ an admissible controlled $\varepsilon$-contractive set of complexity $q^{*}$.

Additional linear constraints and/or alternative optimization costs can be considered in order to satisfy other design requirements. For example, if an enlargement of the polytope $\mathcal{Q}(\mathcal{V})=$ $\operatorname{conv}\left(v^{1}, \ldots, v^{q}\right)$ to a specific direction of the state space is desired, this specification can be achieved by considering an additional linear constraint which defines the regions where the new vertex must be situated. Thus, if the new vertex $v^{*}$ is desired or required to be located in a half

\footnotetext{
${ }^{1}$ For the continuous-time case, from relations (34) and (35) it follows that $p_{q+1}^{*} \leq 0$. For the discrete-time case, it follows that $p_{q+1}^{*} \geq 0$ directly from (40).
} 
space defined by the inequality

$$
g^{\top} x \geq 1
$$

where $g \in \mathrm{R}^{n}$, the inequality $g^{\top} v^{*} \geq 1$ must be considered as an additional linear constraint of the optimization problem. For this particular case, solving the constrained optimization problem (33)-(38) for the continuous-time case or (39)-(44) for the discrete-time case with

$$
\max _{v^{*}, u^{*}, p_{q}^{*}, p_{q+1}^{*}}\left\{g^{\top} v^{*}\right\}
$$

as optimization criterion, we obtain an enlarged admissible controlled $\varepsilon$-contractive set $\mathcal{Q}\left(\mathcal{V}^{*}\right)$ of complexity $q^{*}$ with the new vertex $v^{*}$ belonging to the half space defined by the inequality $g^{\top} v^{*} \geq 1$ and located as far as possible from the boundary $g^{\top} v=1$.

Example 4.3 The results established in Theorem 4.2 can be utilized to develop an algorithmic procedure that produces a monotonically increasing sequence $\left\{\mathcal{X}_{i}\right\}$ of polytopic sets of bounded complexity $q_{\mathcal{X}_{i}} \leq q_{\max }$. Consider the discrete-time system (2) under state and input constraints (5) and (6) respectively. Let $\mathcal{P}\left(G_{i}\right)=\left\{\begin{array}{lll}x \in \mathrm{R}^{2}: & G_{i} x \leq e_{p_{i}}\end{array}\right\}, G_{i}=\left[\begin{array}{llll}g_{i 1} & g_{i 2} & \ldots & g_{i p_{i}}\end{array}\right]^{\top}$ and $\mathcal{Q}\left(\mathcal{V}_{i}\right)=\operatorname{conv}\left(v_{i}^{1}, v_{i}^{2}, \ldots, v_{i}^{q_{i}}\right)$ be the half-space and the vertex representations respectively of the polytopic set $\mathcal{X}_{i}$. Each iteration $i$ of the algorithm consists of the following steps: First, the index sets $\mathcal{N}_{\mathcal{X}_{i}}\left(v^{k}\right), k \in \mathrm{N}_{\left[1, q_{i}\right]}$ as defined in (9), and the polytopic sets $\mathcal{A}_{k}, i \in \mathrm{N}_{\left[1, q_{i}\right]}$ as defined in (10), are calculated. Next, for every non-empty set $\mathcal{A}_{k} \cap \mathcal{P}\left(G_{x}\right)$, where $\mathcal{P}\left(G_{x}\right)$ denotes the state constraint set, the optimization problem

$$
\max _{v^{*}, u^{*}, p_{q}^{*}, p_{q+1}^{*}}\left\{g_{i l}^{\top} v^{*}\right\}
$$

under constraints (39)-(44) is solved. In this formulation, $g_{i l}^{\top}, l \in \mathrm{N}_{\left[1, p_{i}\right]}$, is a row of the matrix $G_{i}$, such that the set $\left\{x \in \mathrm{R}^{n}: g_{i l}^{\top} x \geq 1\right\} \cap \mathcal{A}_{k}$ is nonempty ${ }^{1}$. If a feasible solution exists, then the new set $\mathcal{X}_{i+1}=\operatorname{conv}\left(v_{i}^{1}, v_{i}^{2}, \ldots, v_{i}^{q_{i}}, v^{*}\right)$ is computed. If no solution exists, and in addition, the complexity of the set $\mathcal{X}_{i}$ is less than the specified complexity, i.e., $q_{\mathcal{X}_{i}} \leq q_{\max }$, then the problem (46) under constraints (39)-(43) is solved for $p_{i}$ instances, setting each time the direction $g$ to be equal to $g_{i l}, l \in \mathrm{N}_{\left[1, p_{i}\right]}$. Then, if there exist $l^{*}$ solutions $v_{s}^{*} \notin \mathcal{X}_{i}, s \in \mathrm{N}_{\left[1, l^{*}\right]}$, a new set $\mathcal{X}_{i+1}=\operatorname{conv}\left(v_{i}^{1}, v_{i}^{2}, \ldots, v_{i}^{q_{i}}, v_{\bar{s}}\right)$ is computed, with $\bar{s}:=\arg \max _{s}\left\{g_{i s}^{\top} v_{s}^{*}: s \in \mathrm{N}_{\left[1, l^{*}\right]}\right\}$. If such solutions do not exist, or the complexity of $\mathcal{X}_{i}$ is equal to the specified complexity, the algorithm is terminated.

The procedure described above was applied to a benchmark discrete-time non-autonomous system, namely, the constrained discretized double integrator. The system is described by the state equation (2) with

$$
A=\left[\begin{array}{cc}
1 & T_{s} \\
0 & 1
\end{array}\right], B=\left[\begin{array}{c}
\frac{T_{s}^{2}}{2} \\
T_{s}
\end{array}\right]
$$

where $T_{s}=0.1 \mathrm{sec}$ is the sampling period. The system is subject to hard state and input constraints (5) and (6) respectively, with

$$
G_{x}=\left[\begin{array}{cc}
25^{-1} & 0 \\
0 & 5^{-1} \\
-25^{-1} & 0 \\
0 & -5^{-1}
\end{array}\right], G_{u}=\left[\begin{array}{c}
1 \\
-1
\end{array}\right] .
$$

The objective is to compute an admissible controlled invariant polytopic set of a non-trivial size, but with a complexity that does not exceed a prespecified value.

\footnotetext{
${ }^{1}$ Such an index $l \in \mathrm{N}_{\left[1, p_{i}\right]}$ always exists, since $\mathcal{X}_{i}$ is a full-dimensional polytopic set, thus the vectors $g_{i l}$ span $\mathrm{R}^{n}$.
} 
For comparison purposes, we applied to the same system the method described in (Gutman and Cwikel 1987) for the computation of the largest controlled invariant set. This method is based on the computation of a sequence $\left\{\mathcal{Y}_{i}\right\}$ of monotonically increasing controlled invariant sets $\mathcal{Y}_{i}$, by applying the one-step backward reachability mapping and starting from the singleton set $\mathcal{Y}_{0}:=\{0\}$. In order to be able to evaluate the performance of the proposed approach, the starting set $\mathcal{X}_{0}$ of the proposed method was chosen equal to $\mathcal{Y}_{2}$, which is an admissible controlled invariant full-dimensional set (Cwikel and Gutman 1986). Using the proposed approach, an admissible

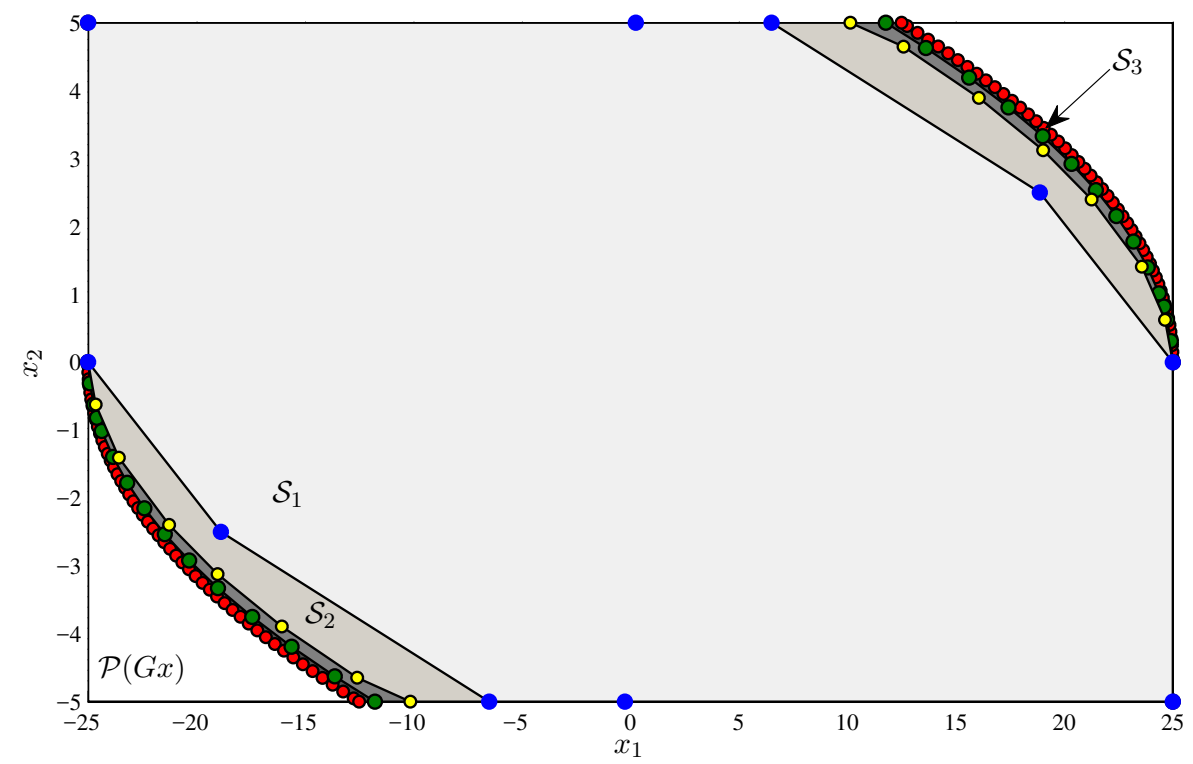

Figure 2. The state constraint set $\mathcal{P}\left(G_{x}\right)$, the maximal controlled invariant set $\mathcal{S}_{\max }$ (106 vertices, in red) and the sets $\mathcal{S}_{1}=\mathcal{X}_{11}$ (10 vertices, in blue), $\mathcal{S}_{2}$ (18 vertices, in yellow), $\mathcal{S}_{3}$ (32 vertices, in green).
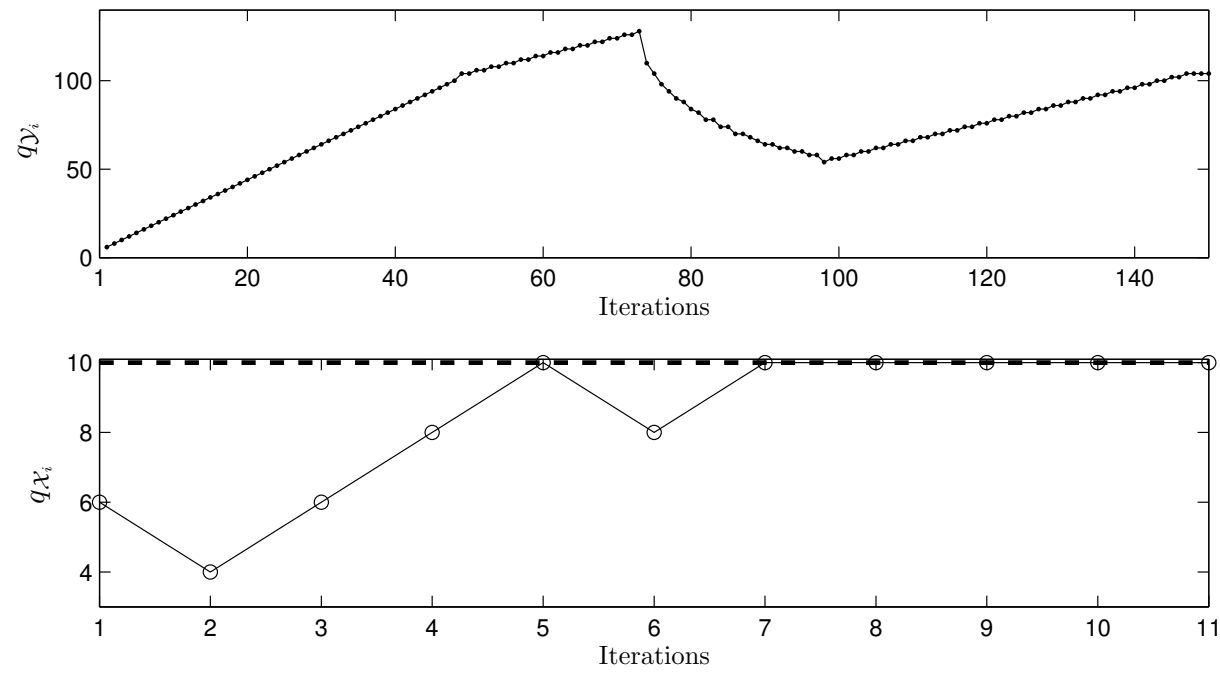

Figure 3. Complexity of the sets produced applying the one-step backward reachability map (Gutman and Cwikel 1987) (upper part), and the sets produced by applying the method proposed in this article (lower plot), for $q_{\max }=10$.

controlled invariant polytope of complexity $\hat{q}_{1}=10$ was obtained at the 11 th iteration. This set, denoted by $\mathcal{S}_{1}:=\mathcal{X}_{11}$, covers $92 \%$ of the surface of the maximal admissible controlled invariant 


\begin{tabular}{lcccc} 
Table $1 . \quad$ Complexity and set coverage for the computed sets. \\
\hline Example 4.3 & $\mathcal{S}_{1}$ & $\mathcal{S}_{2}$ & $\mathcal{S}_{3}$ & $\mathcal{S}_{\max }$ \\
\hline Complexity & 10 & 18 & 32 & 106 \\
Coverage of the set $\mathcal{S}_{\max }(\%)$ & 92 & 98 & 99.63 & 100 \\
\hline
\end{tabular}

set $\mathcal{S}_{\max }$, which has complexity $q_{\mathcal{S}_{\max }}=106$. The maximal admissible controlled invariant set $\mathcal{S}_{\max }$ was reached applying the algorithm in (Gutman and Cwikel 1987) after 148 iterations, i.e., $\mathcal{S}_{\max }=\mathcal{Y}_{148}$. Moreover, the proposed algorithm was also applied for specified complexities of $\hat{q_{2}}=18$ and $\hat{q_{3}}=32$ vertices. The algorithm converged to the admissible controlled invariant sets $\mathcal{S}_{2}$ and $\mathcal{S}_{3}$ correspondingly, which covered $98.1 \%$ and $99.63 \%$ of the surface of the maximal admissible controlled invariant set respectively. The results are summarized in Table 1.

The resulting sets $\mathcal{S}_{1}, \mathcal{S}_{2}$ and $\mathcal{S}_{3}$ along with the state constraint set $\mathcal{P}\left(G_{x}\right)$ and the maximal admissible controlled invariant set are shown in Figure 2.

In Figure 3, the complexity of the sequences $\left\{\mathcal{Y}_{i}\right\},\left\{\mathcal{X}_{i}\right\}$ is shown as functions of the iteration instances of the corresponding algorithmic procedures. In the lower plot, the dotted line corresponds to the complexity constraints set by the problem specifications.

Example 4.4 We consider the benchmark example of the constrained continuous-time triple integrator. The system matrices of system (1) are

$$
A=\left[\begin{array}{lll}
0 & 1 & 0 \\
0 & 0 & 1 \\
0 & 0 & 0
\end{array}\right], B=\left[\begin{array}{l}
0 \\
0 \\
1
\end{array}\right]
$$

We consider box input and state constraints (5) and (6) respectively, with

$$
G_{x}=\left[\begin{array}{ccc}
20^{-1} & 0 & 0 \\
0 & 3^{-1} & 0 \\
0 & 0 & 1 \\
-20^{-1} & 0 & 0 \\
0 & -3^{-1} & 0 \\
0 & 0 & -1
\end{array}\right], G_{u}=\left[\begin{array}{c}
0.5^{-1} \\
-0.5^{-1}
\end{array}\right]
$$

Similarly to Example 4.3, the objective is to construct an admissible controlled invariant polytopic set of a non trivial size, whose complexity does not exceed a value $q_{\max }$. It is worth noticing that for continuous-time systems, a typical method for computing admissible controlled invariant and controlled $\varepsilon$-contractive sets is to compute an invariant approximation by computing the maximal controlled invariant or controlled $\varepsilon$-contractive set of the Euler Auxiliary System, i.e.,

$$
x(t+1)=\left(I_{n \times n}+\tau A\right) x(t)+\tau B u(t)
$$

(see e.g. (Blanchini and Miani 2008) and the references therein for the continuous-time case). To this end, setting $\tau=0.3$ and applying the approach in (Blanchini 1994), the maximal controlled invariant set $\mathcal{S}_{E A S}$ for system (47) was computed, having a complexity of 356 vertices. The large number of vertices is due to the fact that the maximal controlled invariant set $\mathcal{S}_{\max }$ of the continuous-time system, although convex, is not a polytope. A local description of the boundary of the maximal controlled invariant set with curves for general continuous-time systems has been recently established in (De Dona and Levine 2013).

The algorithmic procedure described in Example 4.3 was applied directly to the continuoustime system, with the obvious modification of changing the optimization constraints of the optimization problem solved to relations (33)-(38). Five sets $\mathcal{S}_{i}, i=1,2, \ldots, 5$ were constructed by applying the algorithmic procedure, for different corresponding specified complexities of 10, $16,24,44$, and 62 . The produced sets were found to cover $38 \%, 45 \%, 73.5 \%, 90 \%$ and $94 \%$ of the volume of the maximal admissible controlled invariant set $\mathcal{S}_{E A S}$ of the Euler Auxiliary System respectively. The results are summarized in Table 2. In Figure 4, the constraint set $\mathcal{P}\left(G_{x}\right)$, along 


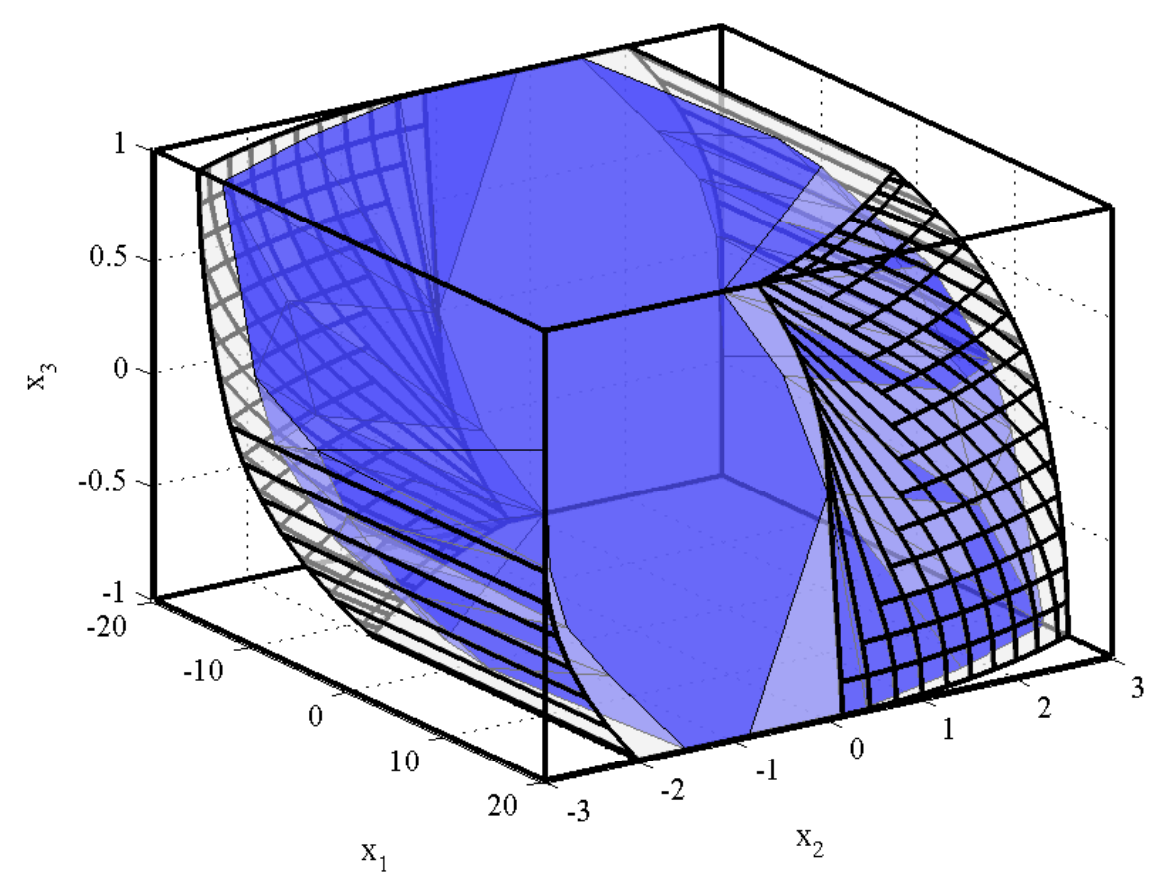

Figure 4. The state constraint set $\mathcal{P}\left(G_{x}\right)$ (white box), the maximal controlled invariant set $\mathcal{S}_{E A S}$ for system (47) setting $\tau=0.3$ (356 vertices, white) and the set $\mathcal{S}_{4}$ (44 vertices, blue) that covers $90 \%$ of the volume of $\mathcal{S}_{E A S}$.

\begin{tabular}{lcccccc} 
Table 2. Complexity and set coverage for the computed sets. \\
\hline Example 4.4 & $\mathcal{S}_{1}$ & $\mathcal{S}_{2}$ & $\mathcal{S}_{3}$ & $\mathcal{S}_{4}$ & $\mathcal{S}_{5}$ & $\mathcal{S}_{E A S}$ \\
\hline Complexity & 10 & 16 & 24 & 44 & 62 & 356 \\
Coverage of the set $\mathcal{S}_{E A S}(\%)$ & 38 & 45 & 73.5 & 90 & 94 & 100 \\
\hline
\end{tabular}

with the sets $\mathcal{S}_{E A S}$ and $\mathcal{S}_{4}$ are shown.

\section{Concluding remarks}

Different from typical existing approaches, in this article the size as well as the complexity were considered as principal design goals for the construction of admissible controlled $\varepsilon$-contractive sets. First, neglecting any invariance property, conditions for enlargement of a polytope by adding a new vertex, such that the enlarged polytope has a prespecified complexity, have been established. Second, conditions for this enlargement approach to preserve the properties of controlled $\varepsilon$-contractiveness have been presented. Finally, by combining these algebraic necessary and sufficient conditions, enlargement methods that produce admissible controlled invariant sets with specified complexity were developed. Generally, the determination of the enlarged admissible sets can be done by solving a series of linear programming problems. It is worth noting that additional performance objectives or other specifications can be taken into account by appropriately adding linear constraints in the relevant optimization problem. The results established here can be extended, under modifications, to the case of linear systems with multiplicative and additive structured uncertainties. While in the two illustrative examples an algorithm for producing convergent monotonically increasing sequences of controlled invariant sets has been presented, it should be emphasized that the goal of this paper is not the development of design algorithms, but merely the establishment of the theoretical background the development of such algorithms can be based upon. 


\section{Acknowledgements}

N. Athanasopoulos gratefully acknowledges the support of the People Programme (Marie Curie Actions) of the European Unions Seventh Framework Programme (FP7/2007-2013 under REA grant agreement no 302345).

\section{References}

Athanasopoulos, N. (2010), "Stability analysis and control of linear and nonlinear constrained systems via Polyhedral Lyapunov functions (in Greek)," University of Patras.

Athanasopoulos, N., and Bitsoris, G. (2010), "Invariant set computation for constrained uncertain discrete-time linear systems," in 49th IEEE Conference on Decision and Control, Atlanta, GA, pp. 5227-5232.

Athanasopoulos, N., Lazar, M., and Bitsoris, G. (2012), "On the Construction of Invariant Proper $\mathcal{C}$-polytopic Sets for Continuous-time Linear Systems," in 16th International Conference on System Theory, Control and Computing, Sinaia, Romania, pp. 1-6.

Aubin, J.P., Viability theory, Systems \& Control: Foundations \& Applications, Birkhaüser (1991).

Benlaoukli, H., and Olaru, S. (2009), "Model Predictive Control - Numerical Methods for the Invariant Sets Approximation," Lecture Notes in Computer Science, Numerical Analysis and Its Applications, 5434, 192-199.

Bertsekas, D.P. (1972), "Infinite-Time Reachability of State-Space Regions by Using Feedback Control," IEEE Transactions on Automatic Control, 17, 604-613.

Bitsoris, G. (1988), "Positively invariant polyhedral sets of discrete-time linear systems," International Journal of Control, 47, 1713-1726.

Bitsoris, G. (1991), "Existence of positively invariant polyhedral sets for continuous-time linear systems," Control Theory and advanced technology, 7, 407-427.

Bitsoris, G. (2013), "Enlargement of Polytopes with Guaranteed Complexity," Technical report, Department of Electrical and Computer Engineering, University of Patras, Greece, CSL-1307.

Blanchini, F. (1990), "Feedback Control for Linear Time-Invariant Systems with State and Control Bounds in the Presence of Disturbances," IEEE Transactions on Automatic Control, 35, $1231-1234$.

Blanchini, F. (1994), "Ultimate boundedness control for uncertain discrete-time systems via set-induced Lyapunov functions," IEEE Transactions on Automatic Control, 39, 428-433.

Blanchini, F., and Miani, S., Set-Theoretic Methods in Control, Systems \& Control: Foundations \& Applications, Boston, Basel, Berlin: Birkhauser (2008).

Blanchini, F., Miani, S., Dorea, C.E.T., and Hennet, J.C. (1999), "Discussion on: (A,B)invariance conditions of polyhedral domains for continuous-time systems," European Journal of Control, 5, 70-81.

Blanchini, F., Miani, S., and Savorgnan, C. (2008), "Dynamic augmentation and complexity reduction of set-based constrained control," in IFAC World Congress, Seoul, South Korea, pp. 14324-14329.

Blanco, T.B., Cannon, M., and De Moor, B. (2010), "On efficient computation of low-complexity controlled invariant sets for unertain linear systems," International Journal of Control, 83, $1339-1446$.

Bobyleva, O.N., and Pyatnitskii, E.S. (2001), "Piecewise-linear Lyapunov functions and localization of spectra of stable matrices," Automation and Remote Control, 62, 1417-1427, Translated from Avtomatika i Telemekhanika, No. 9, 2001, pp. 25-36.

Cannon, M., Deshmukh, V., and Kouvaritakis, B. (2003), "Nonlinear model predictive control with polytopic uncertainties," Automatica, 39, 1487-1494.

Cwikel, M., and Gutman, P.O. (1986), "Convergence of an algorithm to find maximal state constraint sets for discrete-time linear dynamical systems with bounded controls and states," IEEE Transactions on Automatic Control, 31, 457-459. 
De Dona, J.A., and Levine, J. (2013), "On barriers in state and input constrained nonlinear systems," SIAM Journal on Control and Optimizaiton, 51, 3208-3234.

Dórea, C.E.T., and Hennet, J.C. (1999), "(A,B)-Invariant polyhedral sets of linear discrete-time systems," Journal of Optimization Theory and Applications, 103, 521-542.

Gilbert, E.G., and Tan, K.T. (1991), "Linear systems with state and control constraints: the theory and application of maximal output admissible sets," IEEE Transactions on Automatic Control, 36, 1008-1020.

Gutman, P.O., and Cwikel, M. (1987), "An algorithm to find maximal state constraint sets for discrete-time linear dynamical systems with bounded controls and states," IEEE Transactions on Automatic Control, AC-32, 251-254.

Hennet, J.C. (1995), "Discrete Time Constrained Linear Systems," Control and Dynamic Systems, C.T. Leondes Ed., Academic Press, 71, 407-427.

Kolmanovsky, I. V. and Gilbert, E. G., (1998), "Theory and Computation of Disturbance Invariant Sets for Discrete-Time Linear Systems," Mathematical Problems in Egineering, 4, $317-367$.

Lazar, M. (2010), "On infinity norms as Lyapunov functions: Alternative necessary and sufficient conditions," in 49th IEEE Conference on Decision and Control, Atlanta, GA, pp. 5936-5942.

Molchanov, A.P., and Pyatnitskii, E.S. (1986a), "Lyapunov functions that specify necessary and sufficient conditions for absolute stability of nonlinear nonstationary control systems I," Autom. Remote Control, 47, 344-354.

Molchanov, A.P., and Pyatnitskii, E.S. (1986b), "Lyapunov functions that specify necessary and sufficient conditions for absolute stability of nonlinear nonstationary control systems II," Autom. Remote Control, 47, 443-451.

Molchanov, A.P., and Pyatnitskii, E.S. (1986c), "Lyapunov functions that specify necessary and sufficient conditions for absolute stability of nonlinear nonstationary control systems III," Autom. Remote Control, 47, 620-630.

Pluymers, B., Rossiter, J.A., Suykens, J.A.K., and De Moor, B. (2005), "The Efficient Computation of Polyhedral Invariant Sets for Linear Systems with Polytopic Uncerainty," in American Control Conference, Portland, USA, pp. 804-809.

Polański, A. (1995), "On infinity norms as Lyapunov functions for linear systems," IEEE Transactions on Automatic Control, 40, 1270-1273.

Raković, S.V., and Fiacchini, M. (2008), "Invariant Approximations of the Maximal Invariant Set or "Encircling the Square"," in Proceedings of the 17th IFAC World Congress IFAC 2008, Seoul, Korea.

Rohal-Ilkiv, B. (2004), "A note on calculation of polytopic-invariant feasible sets for linear continuous-time systems," Annual reviews in Control, 28, 59-64.

Schrijver, A., Theory of Linear and Integer Programming, Wiley Interscience series in Discrete Mathematics and Optimization (1998).

Scibilia, F. (2010), "Explicit Model Predictive Control: Solutions via Computational Geometry," Norvegian University of Science and Technology.

Scibilia, F., Olaru, S., and Hovd, M. (2011), "On feasible sets for MPC and their approximations.," Automatica, 47, 133-139.

Ziegler, G.M., Lectures on Polytopes, Updated Seventh Printing, Springer (2007).

\section{Appendix A: Proofs}

For proving Theorems 3.1 and 3.2, we first establish necessary and sufficient conditions for a set $\mathcal{Q}\left(\mathcal{V}^{*}\right)=\operatorname{conv}\left(v^{1}, \ldots, v^{q}, v^{*}\right)$ resulting from a set $\mathcal{Q}\left(\mathcal{V}^{0}\right)=\operatorname{conv}\left(v^{1}, \ldots, v^{q}, v^{0}\right)$ by replacement of one vertex $v^{0}$ by $v^{*}$ to satisfy the set relation $\mathcal{Q}\left(\mathcal{V}^{0}\right) \subset \mathcal{Q}\left(\mathcal{V}^{*}\right)$. The following Lemma is a special case of a more general result relative to the enlargement of polytopes (Bitsoris 2013). 
Lemma A.1: Let

$$
\mathcal{Q}\left(\mathcal{V}^{0}\right)=\operatorname{conv}\left(v^{1}, \ldots, v^{q}, v^{0}\right)
$$

be a polytope with vertices $v^{1}, \ldots, v^{q}, v^{0}$ and half-space representation

$$
\mathcal{P}(G)=\left\{x \in \mathrm{R}^{n}: G x \leq e_{s}\right\} .
$$

Then, the set

$$
\mathcal{Q}\left(\mathcal{V}^{*}\right)=\operatorname{conv}\left(v^{1}, \ldots, v^{q}, v^{*}\right)
$$

satisfies the relation

$$
\mathcal{Q}\left(\mathcal{V}^{0}\right) \subset \mathcal{Q}\left(\mathcal{V}^{*}\right)
$$

if and only if the vector $v^{*} \in \mathrm{R}^{n}, v^{*} \neq v^{0}$, satisfies the relation

$$
g_{i}^{\top} v^{*} \geq 1 \quad \forall i \in N_{\mathcal{S}}\left(v^{0}\right) .
$$

Proof: a) Sufficiency: Let $g_{l_{i}}^{\top} x=1, l_{i} \in N_{\mathcal{S}}\left(v^{0}\right) \quad i=1,2, \ldots, s_{0}$ be the faces of the polytope $\mathcal{Q}\left(\mathcal{V}^{0}\right)$ that pass through the vertex $v^{0}$. Setting

$$
G^{0}=\left[\begin{array}{c}
g_{l_{1}}^{\top} \\
g_{l_{2}}^{\top} \\
\vdots \\
g_{l_{s_{0}}}^{\top}
\end{array}\right],
$$

we get

$$
G^{0} v^{0}=e_{s_{0}}
$$

and, by hypothesis (A2), it holds that

$$
G^{0} v^{*}=1+a^{*}
$$

where $a^{*}=\left[\begin{array}{llll}a_{1}^{*} & a_{2}^{*} \cdots & a_{s_{0}}^{*}\end{array}\right]^{\top}$ is a vector with nonnegative components and $a^{*} \neq 0_{s_{0} \times 1}$.

For each face $g_{l_{i}}^{\top} x=1 i=1,2, \ldots, s_{0}$ there exists at least one vertex $v^{k}$ situated on it, i.e., $g_{l_{i}}^{\top} v^{k}=1$ but not on the face $g_{l_{i+1}}^{\top} x=1$, i.e., $g_{l_{i}}^{\top} v^{k}<1$. To simplify the notation, we assume that these are the vertices $v^{i} i=1,2, \ldots, s_{0}$ of the polytope $\mathcal{Q}\left(\mathcal{V}^{0}\right)$. Then, by hypotheses,

$$
G^{0} v^{i}=\left[\begin{array}{c}
g_{l_{1}}^{\top} v^{i} \\
\vdots \\
g_{l_{i_{1}}}^{\top} v^{i} \\
g_{l_{i}}^{\top} v^{i} \\
g_{l_{i+1}}^{\top} v^{i} \\
g_{l_{i+2}}^{\top} v^{i} \\
\vdots \\
g_{l_{s_{0}}}^{\top} v^{i}
\end{array}\right]=\left[\begin{array}{c}
1-a_{i 1} \\
\vdots \\
1-a_{i 2} \\
1 \\
1-a_{i(i+1)} \\
1-a_{i(i+2)} \\
\vdots \\
1-a_{i s_{0}}
\end{array}\right] \quad i=1,2, \ldots, s_{0}
$$


with

$$
\begin{gathered}
a_{i j} \geq 0 \quad i, j=1,2, \ldots, s_{0} \quad j \neq i, i+1, \\
a_{i(i+1)}>0 .
\end{gathered}
$$

We shall prove that

$$
\operatorname{conv}\left(v^{1}, \ldots, v^{s_{0}}, v^{0}\right) \subset \operatorname{conv}\left(v^{1}, \ldots, v^{s_{0}}, v^{*}\right)
$$

To this end, it is sufficient to prove that there exist nonnegative real numbers $\varepsilon^{*}$ and $\varepsilon_{i}$ $i=1,2, \ldots, s_{0}$ such that $\sum_{i=1}^{s_{0}} \varepsilon_{i}+\varepsilon^{*}=1$, and

$$
v^{0}=\varepsilon_{1} v^{1}+\varepsilon_{2} v^{2}+\ldots+\varepsilon_{s_{0}} v^{s_{0}}+\varepsilon^{*} v^{*}
$$

or, equivalently,

$$
G^{0} v^{0}=\varepsilon_{1} G^{0} v^{1}+\varepsilon_{2} G^{0} v^{2}+\ldots+\varepsilon_{s_{0}} G^{0} v^{s_{0}}+\varepsilon^{*} G^{0} v^{*}
$$

because $\operatorname{rank} G^{0}=n$. Setting

$$
a^{i}=\left[\begin{array}{llll}
a_{i 1} & a_{i 2} & \cdots & a_{i s_{0}}
\end{array}\right]^{\top}
$$

and taking into account (A4)-(A6), the relation (A9) can be equivalently written as

$$
e_{s_{0}}=\varepsilon_{1}\left(e_{s_{0}}-a^{1}\right)+\varepsilon_{2}\left(e_{s_{0}}-a^{2}\right)+\ldots+\varepsilon_{s_{0}}\left(e_{s_{0}}-a^{s_{0}}\right)+\varepsilon^{*}\left(e_{s_{0}}+a^{*}\right) .
$$

Thus, it is sufficient to prove that there exist nonnegative real numbers $\varepsilon^{*}$ and $\varepsilon_{i} i=1,2, \ldots, s_{0}$ such that $\sum_{i=1}^{s_{0}} \varepsilon_{i}+\varepsilon^{*}=1$, and

$$
0_{s_{0} \times 1}=\varepsilon_{1} a^{1}+\varepsilon_{2} a^{2} \ldots+\varepsilon_{s_{0}} a^{s_{0}}+\varepsilon^{*} a^{*} .
$$

Such numbers exist because, if

$$
\begin{aligned}
a_{i(i+1)} & >0, \quad i=1,2, \ldots, s_{0}-1, \\
a_{s_{0} 1} & >0, \\
a_{i j} & \geq 0 \quad i, j=1,2, \ldots, s_{0} \quad j \neq i, i+1 \\
a^{*} & >0
\end{aligned}
$$

then the origin is a point of the polytope with vertices

$$
\left[\begin{array}{c}
0 \\
-a_{12} \\
-a_{13} \\
\vdots \\
-a_{i s_{0}}
\end{array}\right],\left[\begin{array}{c}
-a_{21} \\
0 \\
-a_{23} \\
\vdots \\
-a_{i s_{0}}
\end{array}\right], \ldots,\left[\begin{array}{c}
-a_{s_{0} 1} \\
-a_{s_{0} 2} \\
-a_{s_{0} 3} \\
\vdots \\
0
\end{array}\right],\left[\begin{array}{c}
a_{1}^{*} \\
a_{1}^{*} \\
a_{3}^{*} \\
\vdots \\
a_{s_{0}}^{*}
\end{array}\right]
$$


b) Necessity: Let $v^{k_{1}}, v^{k_{2}} \ldots, v^{k_{s *}}$, be the vertices of the set $\mathcal{Q}\left(\mathcal{V}^{*}\right)$ situated on its faces passing through the vertex $v^{*}$. Then,

$$
\begin{aligned}
\mathcal{Q}\left(\mathcal{V}^{*}\right)= & \operatorname{conv}\left(v^{1}, \ldots, v^{s_{0}}, v^{*}\right)= \\
& \operatorname{conv}\left(v^{k_{1}}, v^{k_{2}}, \ldots, v^{k_{s *}}, v^{*}\right) \cup \operatorname{conv}\left(v^{1}, \ldots, v^{s_{0}}\right) .
\end{aligned}
$$

If $\mathcal{Q}\left(\mathcal{V}^{0}\right) \subset \mathcal{Q}\left(\mathcal{V}^{*}\right)$ is satisfied, then $v^{0} \in \operatorname{conv}\left(v^{1}, v^{2}, \ldots, v^{s_{0}}, v^{*}\right)$ but $v^{0} \notin \operatorname{conv}\left(v^{1}, v^{2}, \ldots, v^{s_{0}}\right)$ because otherwise $v^{0}$ would be redundant for the definition of the set $\mathcal{Q}\left(\mathcal{V}^{0}\right)$. Therefore, by (A10), it holds that

$$
v^{0} \in \operatorname{conv}\left(v^{k_{1}}, v^{k_{2}}, \ldots, v^{k_{s *}}, v^{*}\right)
$$

Relation (A11) implies the existence of nonnegative real numbers $\varepsilon_{k_{1}}, \varepsilon_{k_{2}}, \ldots, \varepsilon_{k_{s *}}$ and $\varepsilon^{*}$ such that $\varepsilon_{k_{1}}+\varepsilon_{k_{2}}+\ldots+\varepsilon_{k_{s *}}+\varepsilon^{*}=1$ and

$$
v^{0}=\varepsilon_{k_{1}} v^{k_{1}}+\varepsilon_{k_{2}} v^{k_{2}} \ldots,+\varepsilon_{k_{s *}} v^{k_{s *}}+\varepsilon^{*} v^{*},
$$

or, equivalently,

$$
G^{0} v^{0}=\varepsilon_{k_{1}} G^{0} v^{k_{1}}+\varepsilon_{k_{2}} G^{0} v^{k_{2}}+\ldots+\varepsilon_{k_{s *}} G^{0} v^{k_{s *}}+\varepsilon^{*} G^{0} v^{*}
$$

because the matrix $G^{0}$ defined in (A3) satisfies the relation $\operatorname{rank} G^{0}=n$. Setting

$$
G^{0} v^{*}=e_{s_{0}}+a^{*}
$$

and using the fact that

$$
G^{0} v^{k_{i}}=e_{s_{0}}-a_{k_{i}} \quad \text { with } a_{k_{i}} \geq 0
$$

relation (A12) is equivalently written as

$$
e_{s_{0}}=\varepsilon_{k_{1}}\left(e_{s_{0}}-a^{k_{1}}\right)+\varepsilon_{k_{2}}\left(e_{s_{0}}-a^{k_{2}}\right)+\ldots+\varepsilon_{k_{s *}}\left(e_{s_{0}}-a^{k_{s *}}\right)+\varepsilon^{*}\left(e_{s_{0}}+a^{*}\right),
$$

or

$$
0=-\varepsilon_{k_{1}} a^{k_{1}}-\varepsilon_{k_{2}} a^{k_{2}}-\ldots-\varepsilon_{k_{s *}} a^{k_{s *}}+\varepsilon^{*} a^{*} .
$$

The last relation implies that $a^{*} \geq 0$. Therefore,

$$
g_{i}^{\top} v^{*}>1, \quad \forall i \in N_{\mathcal{S}}\left(v^{0}\right)
$$

In addition, $v^{*} \notin \mathcal{Q}\left(\mathcal{V}^{0}\right)$ because otherwise $\mathcal{Q}\left(\mathcal{V}^{0}\right)=\mathcal{Q}\left(\mathcal{V}^{*}\right)$.

\section{A.1 Proof of Theorem 3.1}

a) Sufficiency: If $v^{*} \notin \mathcal{Q}(\mathcal{V})$ and $v^{*} \in \mathcal{A}_{1} \cup \mathcal{A}_{2} \cup \ldots \cup \mathcal{A}_{q}$ then there exists an index $k \in N_{[1, q]}$ such that $v^{*} \in \mathcal{A}_{k}$. By definition (10), this yields

$$
g_{j}^{\top} v^{*} \geq 1 \quad \forall j \in N_{\mathcal{S}}\left(v^{k}\right)
$$


Therefore,

$$
\mathcal{Q}(\mathcal{V}) \subset \mathcal{Q}\left(\mathcal{V}^{*}\right)
$$

Furthermore, by Lemma A.1, (A16) yields

$$
\operatorname{conv}\left(v^{1}, v^{2}, \ldots, v^{q}\right) \subset \operatorname{conv}\left(v^{1}, v^{2}, \ldots, v^{k-1}, v^{k+1}, \ldots v^{q}, v^{*}\right)
$$

which in turn implies that

$$
\operatorname{conv}\left(v^{1}, v^{2}, \ldots, v^{k-1}, v^{k+1}, \ldots, v^{q}, v^{*}\right)=\operatorname{conv}\left(v^{1}, v^{2}, \ldots, v^{q}, v^{*}\right) .
$$

Therefore, the polytope $\mathcal{Q}\left(\mathcal{V}^{*}\right)$ is of equal or lower complexity than $\mathcal{Q}(\mathcal{V})$.

b) Necessity: If $\mathcal{Q}(\mathcal{V}) \subset \mathcal{Q}\left(\mathcal{V}^{*}\right)$, that is

$$
\operatorname{conv}\left(v^{1}, v^{2}, \ldots, v^{v^{q}}\right) \subset \operatorname{conv}\left(v^{1}, v^{2}, \ldots, v^{q}, v^{*}\right)
$$

then $v^{*} \notin \mathcal{Q}(\mathcal{V})$. If, in addition, the polytope $\mathcal{Q}\left(\mathcal{V}^{*}\right)$ is of equal or of lower complexity, then there exists at least one index $k \in \mathrm{N}_{[1, q]}$ such that the corresponding vertex $v^{k}$ is redundant for the description of set $\mathcal{Q}\left(\mathcal{V}^{*}\right)$. Therefore, relation (A18) can be written as

$$
\begin{aligned}
& \operatorname{conv}\left(v^{1}, v^{2}, \ldots, v^{k-1}, v^{k}, v^{k+1}, \ldots v^{q}\right) \\
& \subset \operatorname{conv}\left(v^{1}, v^{2}, \ldots, v^{k-1}, v^{k+1}, \ldots v^{q}, v^{*}\right) .
\end{aligned}
$$

According to Lemma A.1, this relation implies that

$$
g_{j}^{\top} v^{*} \geq 1, \quad j \in N_{\mathcal{S}}\left(v^{k}\right)
$$

or, equivalently, $v^{*} \in \mathcal{A}_{k .}$. Consequently, $v^{*} \in \mathcal{A}_{1} \cup \mathcal{A}_{2} \ldots \cup \mathcal{A}_{q}$.

\section{A.2 Proof of Theorem 3.2}

a) Sufficiency: From (15), (16) it follows that

$$
v^{*} \in \mathcal{A}_{k_{i}} \text { for } i=1,2, \ldots, l+1 \text {. }
$$

This, by Theorem 3.1, implies that

$$
\begin{aligned}
& \operatorname{conv}\left(v^{1}, v^{2}, \ldots, v^{q}\right) \subset \\
& \operatorname{conv}\left(v^{1}, v^{2}, \ldots, v^{k_{i}-1}, v^{k_{i}+1}, \ldots v^{q}, v^{*}\right)=\mathcal{Q}\left(\mathcal{V}^{*}\right)
\end{aligned}
$$

for $i=1,2, \ldots, l+1$. Consequently, the vectors $v^{k_{i}} i=1,2, \ldots, l+1$ are redundant in the description of $\mathcal{Q}\left(\mathcal{V}^{*}\right)$, thus the polytope $\mathcal{Q}\left(\mathcal{V}^{*}\right)$ is of complexity $q^{*}$ lower or equal to $q^{*}=q-l$.

b) Necessity: If $\mathcal{Q}(\mathcal{V}) \subset \mathcal{Q}\left(\mathcal{V}^{*}\right)$, or equivalently

$$
\operatorname{conv}\left(v^{1}, v^{2}, \ldots, v^{v^{q}}\right) \subset \operatorname{conv}\left(v^{1}, v^{2}, \ldots, v^{q}, v^{*}\right)
$$

then $v^{*} \notin \mathcal{Q}(\mathcal{V})$. If, in addition, the polytope is of complexity $q^{*}$ lower or equal to $q^{*}=q-l$, then there exist at least $l$ indices $k_{i} \in \mathrm{N}_{[1, q]} i=1,2, \ldots, l+1$, such that the corresponding vertices 
$v^{k_{i}}$ are redundant for the description of the set $\mathcal{Q}\left(\mathcal{V}^{*}\right)$. Therefore, from relation (A20) it follows that

$$
\begin{gathered}
\operatorname{conv}\left(v^{1}, v^{2}, \ldots, v^{k_{i}-1}, v^{k_{i}}, v^{k_{i}+1}, \ldots, v^{q}\right) \\
\subset \operatorname{conv}\left(v^{1}, v^{2}, \ldots, v^{k_{i}-1}, v^{k_{i}+1}, \ldots, v^{q}, v^{*}\right),
\end{gathered}
$$

for $i=1,2, \ldots, l+1$. By Lemma A.1, this relation implies that

$$
g_{j}^{\top} v^{*} \geq 1, \quad \forall j \in N_{\mathcal{S}}\left(v^{k_{i}}\right), \quad \forall i=1,2, \ldots, l+1,
$$

or, equivalently, $v^{*} \in \mathcal{A}_{k_{1}} \cap \mathcal{A}_{k_{2}} \cap \ldots \cap \mathcal{A}_{k_{l+1}}$.

\section{A.3 Proof of Theorem 3.5}

If $\mathcal{Q}(\mathcal{V})$ is a controlled $\varepsilon$-contractive set, there exist (Blanchini and Miani 2008, Theorem 4.34) a matrix ${ }^{1} P \in \mathrm{R}^{q \times q}$, such that $p_{i j} \geq 0$, for all $i \in \mathrm{N}_{[1, q]}, j \in \mathrm{N}_{[1, q]}, i \neq j$, and a matrix $U \in \mathrm{R}^{m \times q}$, such that

$$
\begin{aligned}
& A V+B U=V P \\
& e_{q}^{\top} P \leq-\varepsilon e_{q}^{\top},
\end{aligned}
$$

where $V=\left[\begin{array}{lll}v^{1} & \ldots & v^{q}\end{array}\right]$. Let $P^{\star} \in \mathrm{R}^{(q+1) \times(q+1)}, V^{\star} \in \mathrm{R}^{n \times q+1}, U^{\star} \in \mathrm{R}^{m \times q+1}$ be matrices defined as follows

$$
P^{\star}:=\left[\begin{array}{cc}
P & p^{*} \\
0_{1 \times q} & p_{q+1}^{*}
\end{array}\right]
$$

$V^{\star}:=\left[\begin{array}{ll}V & v^{*}\end{array}\right], V^{\star}:=\left[\begin{array}{ll}U & u^{*}\end{array}\right]$. Then, from relations (19)-(21), it follows that conditions (A21),(A22) are also satisfied for $\mathcal{Q}\left(\mathcal{V}^{*}\right)$, with $P=P^{\star}, V=V^{\star}$ and $U=U^{\star}$. Thus, $\mathcal{Q}(\mathcal{V})$ is also a controlled $\varepsilon$-contractive set.

Conversely, if $\mathcal{Q}(\mathcal{V})$ is a controlled $\varepsilon$-contractive set, there exists a matrix $\hat{P} \in \mathrm{R}^{(q+1) \times(q+1)}$, $\hat{p}_{i j} \geq 0$, for all $i \in \mathrm{N}_{[1, q]}, j \in \mathrm{N}_{[1, q]}, i \neq j$, which satisfies conditions (A21), (A22) with $V=V^{\star}$ and $U=U^{\star}$. Then, relations $(19)-(21)$ are satisfied with $p_{i}^{*}=\hat{p}_{(q+1) i}, i=1, \ldots, q$ and $p_{q+1}^{*}=$ $\hat{p}_{(q+1)(q+1)}$.

\section{A.4 Proof of Theorem 3.7}

If $\mathcal{Q}(\mathcal{V})$ is a controlled $\varepsilon-$ contractive set, there exist (Blanchini and Miani 2008, Theorem 4.41) a nonnegative matrix $P \in \mathrm{R}_{+}^{q \times q}$ and a matrix $U \in \mathrm{R}^{m \times q}$, such that

$$
\begin{aligned}
A V+B U & =V P \\
e_{q}^{\top} P & \leq \varepsilon e_{q}^{\top},
\end{aligned}
$$

where $V=\left[\begin{array}{lll}v^{1} & \ldots & v^{q}\end{array}\right]$. where $V=\left[\begin{array}{lll}v^{1} & \ldots & v^{q}\end{array}\right]$. Let $P^{\star} \in \mathrm{R}^{(q+1) \times(q+1)}, V^{\star} \in \mathrm{R}^{n \times q+1}, U^{\star} \in \mathrm{R}^{m \times q+1}$ be matrices defined as follows

$$
P^{\star}:=\left[\begin{array}{cc}
P & p^{*} \\
0_{1 \times q} & p_{q+1}^{*}
\end{array}\right],
$$

\footnotetext{
${ }^{1}$ Matrices having these properties are referred in the literature as $\mathcal{M}$-matrices, Metzler matrices, or essentially nonnegative matrices.
} 
$V^{\star}:=\left[\begin{array}{ll}V & v^{*}\end{array}\right], V^{\star}:=\left[\begin{array}{ll}U & u^{*}\end{array}\right]$. Then, from relations (25)-(28), it follows that conditions (A23),(A24) are also satisfied for $\mathcal{Q}\left(\mathcal{V}^{*}\right)$, with $P=P^{\star}, V=V^{\star}$ and $U=U^{\star}$. Thus, $\mathcal{Q}(\mathcal{V})$ is also a controlled $\varepsilon$-contractive set.

Conversely, if $\mathcal{Q}(\mathcal{V})$ is a controlled $\varepsilon$-contractive set, there exists a nonnegative matrix $\hat{P} \in$ $\mathrm{R}_{+}^{(q+1) \times(q+1)}$ satisfying conditions (A23), (A24) with $V=V^{\star}$ and $U=U^{\star}$. Then, relations $(25)-(28)$ are satisfied with $p_{i}^{*}=\hat{p}_{(q+1) i}, i=1, \ldots, q$ and $p_{q+1}^{*}=\hat{p}_{(q+1)(q+1)}$.

\section{A.5 Proof of Theorem 4.1}

By virtue of Theorem 3.5, relations (33)-(35) are necessary and sufficient for the set $\mathcal{Q}\left(\mathcal{V}^{*}\right)$ to be controlled $\varepsilon$-contractive. Setting $V^{\star}=\left[\begin{array}{ll}V & v^{*}\end{array}\right], U^{\star}=\left[\begin{array}{ll}U & u^{\star}\end{array}\right]$, from the proof of Theorem 3.5, there exists a matrix $P^{\star} \in \mathrm{R}^{(q+1) \times(q+1)}, p_{i j} \geq 0$ for all $i \neq j$, such that $A V^{\star}+B U^{\star}=V^{\star} P^{\star}$ and $e_{q+1}^{\top} P^{\star} \leq-\varepsilon e_{q+1}^{\top}$. Moreover, since $\mathcal{Q}(\mathcal{V})$ is an admissible controlled $\varepsilon$-contractive set, for each vertex $v^{i}, i \in \mathrm{N}_{[1, q]}$ there exist control inputs $u^{i} \in \mathrm{R}^{m}, i \in \mathrm{N}_{[1, q]}$, such that $G_{x} v^{i} \leq e_{p_{x}}$, $G_{u} u^{i} \leq e_{p_{u}}$. Also, from (36), it follows that $\mathcal{Q}\left(\mathcal{V}^{*}\right) \subset \mathcal{P}\left(G_{x}\right)$, where $\mathcal{P}\left(G_{x}\right)$ is the state constraint set (5). Moreover, by (37) and (Blanchini and Miani 2008, Theorem 4.34) it follows that there exists a stabilizing control law $u=f(x)$ such that $f\left(x\left(t ; x_{0}\right) \in \mathcal{P}\left(G_{u}\right)\right.$, for any $x_{0} \in \mathcal{P}\left(G_{x}\right)$, where $\mathcal{P}\left(G_{u}\right)$ is the input constraint set (6). Thus, the set $\mathcal{Q}\left(\mathcal{V}^{*}\right)$ is an admissible controlled $\varepsilon$-contractive set. Finally, from Theorem 3.2 and (38), it follows that there exist indices $k_{i}$, $i=1, \ldots, q-q^{*}+1$ such that $v^{*} \in \mathcal{A}_{k_{1}} \cap \mathcal{A}_{k_{2}} \cap \ldots \cap \mathcal{A}_{k_{q-q^{*}+1}}$. Consequently, the complexity of the set $\mathcal{Q}\left(\mathcal{V}^{\star}\right)$ is $q-\left(q-q^{*}\right)=q^{*}$.

\section{A.6 Proof of Theorem 4.2}

The proof of Theorem 4.2 follows the same steps as in Theorem 4.1 and is omitted. 